\title{
Herida penetrante del tórax por un proyectil de arma de fuego localizado inicialmente en el ventrículo izquierdo y luego en la arteria renal derecha
}

\author{
Ludmila Cosío Lima, ${ }^{1}$ Raúl Gámiz González, ${ }^{2}$ Miguel Cosío Pascal ${ }^{3}$
}

\section{Resumen}

Objetivo: Presentar un caso de herida penetrante del corazón con migración del proyectil y comentar los criterios actuales para el manejo de este tipo de pacientes. Las heridas por proyectil de arma de fuego penetrantes a tórax cada día son más frecuentes en todo el mundo y traen aparejada una mortalidad considerable, ya que el calibre y el poder de las armas también son actualmente más letales. Los heridos que logran llegar vivos al hospital están generalmente supeditados a la atención paramédica-médica precoz en la ambulancia o helicóptero y al tiempo que dure el traslado al hospital. Gracias al personal entrenado para manejar estos problemas en lapsos muy breves y a los adelantos tecnológicos para contribuir a valorar la situación, el pronóstico de estos heridos ha mejorado.

Palabras clave: Heridas de corazón, letalidad, cirugía en los sobrevivientes.

\section{INTRODUCCIÓN}

Las heridas por arma de fuego actualmente son muy frecuentes en todo el mundo. Este fenómeno se debe a múltiples causas, entre ellas a los enormes y múltiples intereses involucrados en su producción, falta de control por las autoridades, impune contrabando, delincuencia

\footnotetext{
1 Associate Professor of Exercise Science. Exercise Science and Community Health, University of West Florida, FL. USA.

2 Cirujano de la Unidad de Gineco-Obstetricia, CMN, IMSS.

${ }^{3}$ Cirujano de Tórax, Hospital Ángeles Mocel.
}

Correspondencia:

Dr. Miguel Cosío-Pascal

Correo electrónico: micopa1311@aol.com

Aceptado: 10-11-2015.

Este artículo puede ser consultado en versión completa en http:// www.medigraphic.com/actamedica

\begin{abstract}
Summary
Purpose: The authors wish to present an original case of a gunshot wound to the left ventricle with bullet migration, as well as to discuss the necessity of adequate medical treatment of such unique cases. Due to the enhanced caliber and power of modern weapons, gunshot wounds to the chest cavity are occurring with greater frequency and fatality worldwide. The survival of those gunshot victims who do make it to the hospital depends on the efficacy of EMT treatment, and rapid emergency response time via ambulance or helicopter transport. With enhanced training of paramedics and emergency response staff who are able to provide quick and effective treatment to these patients, as well as the rise of technological advancements, the prognosis of these victims has improved.
\end{abstract}

Key words: Cardiac wounds, fatalities, surgery in survivors.

organizada, guerras — casi siempre por fanatismo religioso-, guerrillas, etcétera. Con sorpresa y preocupación, nos enteramos de que en varios países las sectas de varias religiones, los adolescentes y aun los niños tienen acceso a armas de grueso calibre, y el mundo se horroriza al difundirse las masacres que tienen lugar en escuelas, iglesias y otros sitios públicos e, inclusive, en los hogares. Hace poco se publicó en la prensa que un niño de tres años tuvo acceso a una pistola, la disparó e hirió a sus padres.

Las primeras menciones acerca de las heridas de corazón se encuentran en un papiro egipcio (Imhotep) que data de 3,000 a. C. (Edwin Smith) y en obras como La Ilíada de Homero. Hasta el siglo IX, los médicos consideraban que estas heridas eran mortales por necesidad. Bilroth advirtió en 1882 que el cirujano que se atreviera a tratar a un herido de corazón quedaría desacreditado de por vida. Sin embargo, pocos años después, en 1896, Rhen reportó el primer caso tratado con éxito de una herida en la aurícula derecha. ${ }^{1,2}$ 


\section{PRESENTACIÓN DEL CASO}

Llegó al hospital, en ambulancia y atendido por paramédicos, un individuo de sexo masculino, de 24 años de edad, con una herida de bala en el hemitórax izquierdo, sin orificio de salida. El paciente se encontraba razonablemente estabilizado, con dos venas canalizadas y oxígeno. Estaba consciente y declaró que su vecino le disparó como a tres metros de distancia por una discusión. Las vías respiratorias estaban permeables, la TA 82/44, con 124 latidos cardiacos por minuto. Las radiografías de tórax localizaron el proyectil en el espesor de la pared del ventrículo izquierdo (Figura 1). El orificio de entrada era nítido y se ubicaba ligeramente arriba de la tetilla izquierda, a cinco centímetros del borde izquierdo del esternón. El sangrado externo no era profuso. Bajo anestesia general, se realizó toracotomía anterolateral a la altura del quinto espacio intercostal. Se aspiraron 80 $\mathrm{mL}$ de sangre de la cavidad pleural. En el lóbulo superior izquierdo del pulmón se identificó la herida, que prácticamente no sangraba y dejaba escapar muy poco aire. El pericardio se encontraba perforado por el proyectil. Existía hemopericardio, pero sin llegar a tamponade, seguramente debido a que el proyectil incrustado en el espesor del ventrículo lo impedía. Se procedió enseguida a abrir el pericardio en toda su longitud, separando los bordes con suturas a tracción con objeto de tener un campo visual operatorio adecuado. La herida de la bala se localizó en la parte alta del ventrículo izquierdo, muy cerca de la arteria coronaria descendente anterior. No era

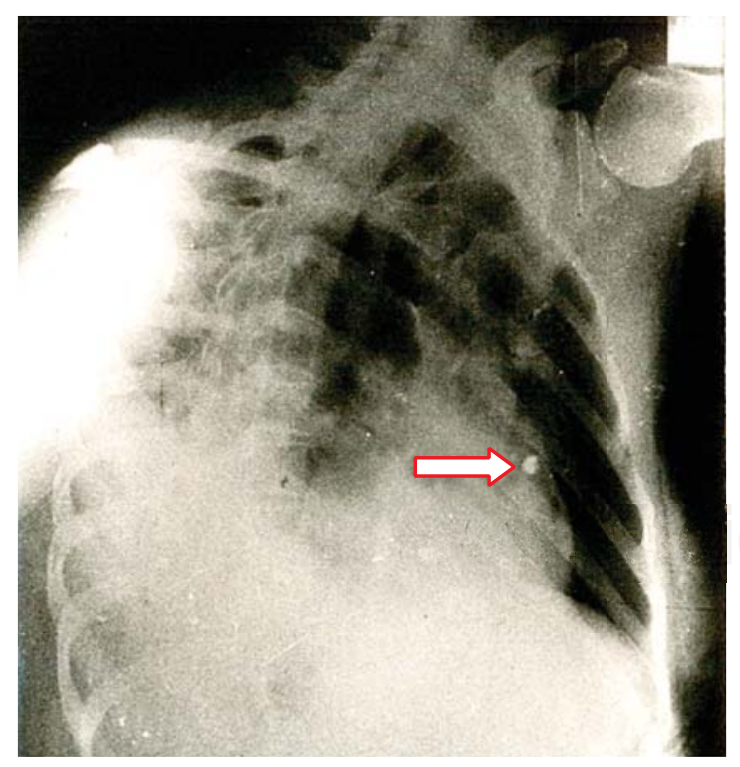

Figura 1. El proyectil se encontraba alojado en el espesor de la parte alta del ventrículo izquierdo. nítida e iba a ser difícil repararla. El proyectil no se visualizó. Tratamos de localizarlo en el espesor del músculo, pero sin lograrlo. Con cada latido del corazón, escapaba escasa cantidad de sangre por la herida. No intentamos emplear una pinza de Allis por temor de ampliar la herida o lesionar la coronaria. En vista de la situación, decidimos explorar el interior del ventrículo a través de la orejuela. La aurícula, válvula mitral y estructuras subvalvulares se encontraron completamente normales, pero la bala no se halló. Solicitamos la presencia de los técnicos en imagenología en el quirófano para tratar de ubicar el proyectil por medio de radiografías. En el tórax, los resultados fueron negativos, pero en la placa de abdomen se visualizó el proyectil a escasa distancia del riñón derecho. Se procedió de inmediato a suturar cuidadosamente la herida cardiaca con Prolene 2-00 con tres puntos en "colchonero" verticales anclados con teflón, tratando de no involucrar a arterias coronarias y cerciorándonos de que no sangraba. La toracotomía se cerró, dejando dos sondas de drenaje sin succión. Acto seguido, se reposicionó al sujeto para realizar la intervención adecuada, con objeto de recuperar la bala. El proyectil, calibre 22, se encontraba en el interior de la arteria renal derecha antes de su división (Figura 2). Se procedió a retirarlo, reparando enseguida el vaso. El herido evolucionó satisfactoriamente. Fue externado a los siete días. Se revisó a las dos semanas y se le observó muy bien.

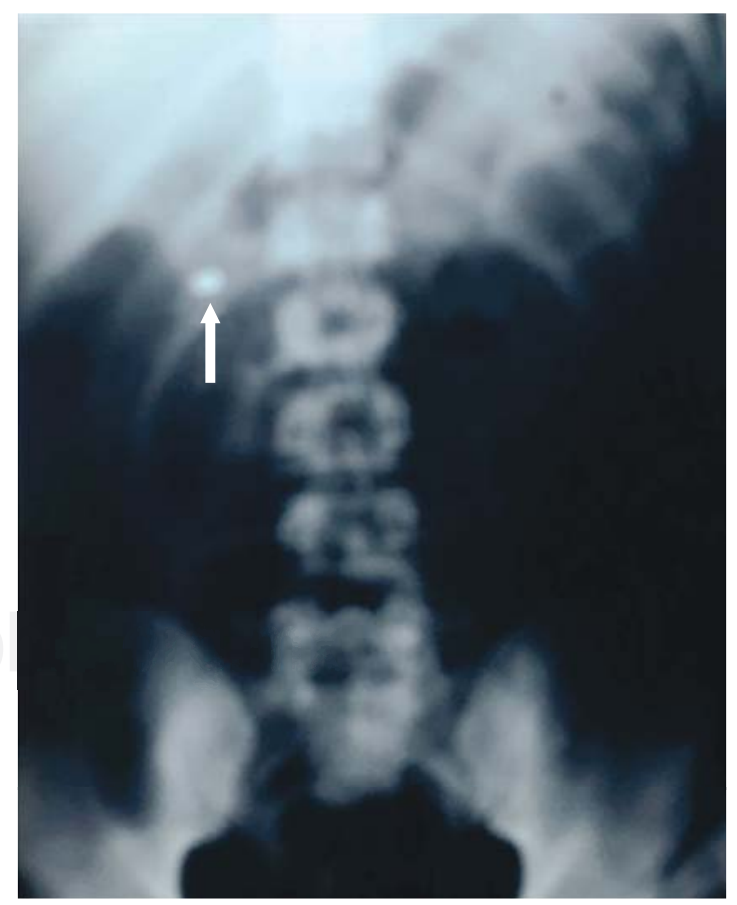

Figura 2. La bala emigró a la arteria renal derecha. 


\section{DISCUSIÓN}

El corazón es un órgano único, que empieza a latir sin descanso a la onceava semana de vida intrauterina y lo sigue haciendo hasta la muerte del individuo. En la actualidad, se calcula que $70 \%$ del total de los latidos de esos corazones que deberían seguir latiendo son anulados prematuramente por una herida en la víscera, que produce la muerte. Las heridas por arma blanca tienden a disminuir, mientras las producidas por armas de fuego van constantemente en aumento.

Las estadísticas en nuestro país no son del todo confiables y la literatura es escasa, pero los ciudadanos nos percatamos perfectamente de la terrible situación. En los EUA, los números son impresionantes. Las heridas del corazón constituyen la primera causa de muerte en individuos entre uno y 48 años, y el 34\% muere antes de llegar al hospital. ${ }^{3}$ Según el FBI, cada 25.3 segundos se comete un crimen violento, lo que significa al año el doble de las muertes por "terrorismo" y el 50\% del total de las bajas durante la guerra de Afganistán. El gobierno norteamericano ha invertido más de medio billón de dólares para combatir el terrorismo, pero para el control y producción de las armas de fuego la voluntad ha sido mínima, además de que muchos ciudadanos se oponen a la medida por su idiosincrasia y son apoyados por organizaciones tan poderosas como la NRA (siglas en inglés de la Asociación Nacional del Rifle). ${ }^{1,2}$

El trayecto del proyectil que entra al tórax es casi imposible de determinar, especialmente en aquellos casos que no tienen orificio de salida.

La migración de los proyectiles que permanecen dentro del cuerpo está descrita desde hace tiempo y existe mucha literatura al respecto. Por lo general, las migraciones son de las venas periféricas al ventrículo derecho o del ventrículo izquierdo hacia arterias, como en nuestro caso. Se ha descrito también la migración del proyectil del ventrículo izquierdo al derecho a través de una comunicación interventricular, con embolismo posterior hacia arterias pulmonares. Así mismo, existen migraciones no relacionadas al sistema cardiovascular, fundamentalmente las de espacios intermusculares. Son pocos los proyectiles que embolizan a vasos cerebrales, pero cuando el proyectil migra hacia alguna arteria mayor de la circulación cerebral, la mortalidad es de $23-37 \%{ }^{4}$

El trauma torácico constituye entre el $25-50 \%$ de todos los traumatismos, y su manejo ha evolucionado mucho desde que Beall ${ }^{5}$ realizó en 1961 la primera toracotomía de urgencia en un individuo con paro cardiaco, con objeto de realizar las maniobras de resucitación directas. El procedimiento es reconocido actualmente por el Colegio Americano de Cirujanos. Con esta técnica, el cirujano puede controlar el sangrado masivo del corazón, el tamponade, e intentar la cardiorrafia, pinzando la aorta descendente con objeto de aumentar el flujo de sangre a las arterias carótidas y coronarias, así como practicar el masaje cardiaco directamente. Para que el procedimiento se realice con mayores probabilidades de éxito, el herido debe ser trasladado desde el lugar en que fue herido al hospital sin rebasar los 10-20 minutos "de oro". El traslado en una ambulancia o helicóptero bien equipados y con personal entrenado que inicie las maniobras de resucitación mejora el pronóstico. Los norteamericanos llaman a este tipo de traslado "scoop and run", algo así como "recógelo, levántalo y corre". En Europa está permitido realizar la toracotomía de urgencia durante el traslado al hospital.

Las heridas penetrantes de corazón complejas son las que dañan estructuras intracardiacas y/o arterias coronarias. Wall ${ }^{6}$ reportó 711 casos tratados durante 20 años, 90\% de ellos era de sexo masculino, con edad promedio de 31.1 ; $54 \%$ heridos por arma blanca y $42 \%$ por arma de fuego. Hubo 60 personas con heridas complejas. La mortalidad por heridas de arterias coronarias, todas tratadas con ligadura, fue de $78.2-52 \%$. En un individuo se realizó un puente aortocoronario en la arteria descendente anterior, sin éxito, pues el paciente también falleció. Llevó a cabo dos cambios de válvula mitral por prótesis en operaciones posteriores y reparó 14 casos de fístulas, con $35.7 \%$ de mortalidad. Los sobrevivientes requirieron de intervenciones posteriores para terminar de reparar las heridas cardiacas complejas.

\section{CONCLUSIÓN}

Las heridas penetrantes de corazón presentan cuadros clínicos muy diversos, ya que dependen de muchos y muy variados factores. En el caso que presentamos intervinieron indudablemente variables positivas, como ser herido con un proyectil de pequeño calibre que no produjo daños importantes en la pared, pulmón o vasos sanguíneos, y habiéndose localizado muy cerca de la coronaria descendente anterior, no la dañó. Además, el proyectil, hasta llegar al hospital, se encontraba en el espesor muscular del ventrículo izquierdo, funcionando como tapón, lo que impidió el sangrado masivo y probable tamponade. La sutura del orificio de entrada al ventrículo no fue fácil de reparar por su cercanía a la coronaria.

Los heridos tratados con toracotomía de urgencia, que se piensa que pueden sobrevivir sin daño neurológico, deben ser muy bien seleccionados por médicos expertos en estos casos, y hay que ser muy estrictos de acuerdo al estado fisiopatológico del herido. ${ }^{7}$ La toracotomía se lleva a cabo ante cualquier signo de vida - aunque existen aún debates en cuanto a la definición del término "signos de vida". Hay mucha literatura y propuestas al respecto, e incluso, acerca de resultados en series practicadas por el mismo equipo. Rhee ${ }^{8}$ revisó 4,600 casos de toracotomía de emergencia con supervivencia de $8 \%$. Pasquale ${ }^{9}$ publicó 
que de 106 sujetos que recibieron el tratamiento durante el traslado, sobrevivieron tres. En los EUA está prohibido practicar la toracotomía durante el traslado al hospital. Millham ${ }^{10}$ señaló cuales serían los factores más determinantes para la supervivencia de los heridos agónicos tratados con toracotomía de urgencia por heridas penetrantes de tórax. Concluimos que el nivel de evidencias definitivas para indicar o contraindicar el procedimiento no existe en forma estandarizada. Unos individuos evolucionan mejor que otros que tenían las mismas posibilidades de acuerdo a la evaluación preoperatoria.

A pesar de la baja tasa de supervivencia, la toracotomía de urgencia se sigue practicando, ya que vale mucho la pena intentar sacar adelante a un herido severamente chocado o agónico.

\section{REFERENCIAS}

1. Gabrielli NM, Muñoz S, Troncoso GP et al. Heridas penetrantes cardiacas. Cuad Cir. 2007; 21: 75-83.
2. Asensio JA, Soto SN, Forno W, Roldan G, Petrone P, Salim A et al. Penetrating cardiac injuries: a complex challenge. Injury. 2001; 32 (7): 533-543.

3. Seamon MJ, Chovanes J, Fox N, Green R, Manis G, Tsiotsias G et al. The use of emergency department for traumatic cardio-pulmonary arrest. Injury. 2012; 43 (9): 1355-1361.

4. Dalsetan MR, Jinkins JR. Peripheral vascular gunshot bullet embolus to the cerebral circulation. Neuroradiology. 1990; 23: 516-519.

5. Beall AC Jr, Ochsner JL, Morris GC Jr, Cooley DA, Debakey ME. Penetrating wounds of the heart. J Trauma. 1961; 1: 195-207.

6. Wall MJ, Mattox KL, Chan CD, Baldwin JC. Acute management of complex cardiac injuries. J Trauma. 1997; 42 (5): 905-912.

7. Lorenz HP, Steinmetz B, Lieberman J, Schecoter WP, Macho JR. Emergency thoracotomy survival correlates with physiological status. J Trauma. 1992; 32: 780-785.

8. Rhee PM, Acosta J, Bridgevan A, Wang D, Jordan M, Rich N. Survival after emergency department thoracotomy: a review of published data from the past 25 years. J Am Coll Surg. 2000; 190: 288-298.

9. Pasquale MD, Rhodes M, Cipolle MD et al. Defining "dead on arrival": impact on a level I trauma center. J Trauma. 1996; 41: 726-730.

10. Millham FH, Grindingler GA. Survival determinants in patients undergoing emergency room thoracotomy for penetrating chest injury. J Trauma. 1993; 34: 332-336. 